\title{
Das Verhalten der Kaliumchromate bei höherer Temperatur.
}

\author{
Von \\ E. Groschurf.
}

Mit 2 Figuren im Text.

In neverer Zeit sind zablreiche Arbeiten über die Chromsäure und die Chromate erschienen. Die meisten beschäftigen sich mit Vorgängen und Zuständen in wässeriger Lösung oder mit dem Verhalten der Salze bei Gegenwart von Wasser. Über das Verhalten der Chromate bei höherer T'emperatur sind wir dagegen fast nicht orientiert. Im besonderen wissen wir noch nichts über die Existenzbedingungen der Polychromate in der Nähe ibrer Schmelztemperaturen. ${ }^{1}$ Mit der von $G$. Tammans ${ }^{2}$ ausgearbeiteten Methode der thermischen Analyse hoffte ich, mich näher über die Existenzgebiete und das Verhalten der Chromate bei höheren Temperaturen orientieren zu können. Mit Rücksicht auf die Erfahrungen, die ich früher $^{3}$ über die Beständigkeit mehrfach saurer Salze machen konnte, wählte ich zur Untersuchung zunächst die Kaliumchromate.

\section{Kaliummonochromat.}

Es ist bekannt, dafs Kaliummonochromat, $\mathrm{K}_{2} \mathrm{CrO}_{4}$, beim Erhitzen seine Farbe unter Dekrepitieren ändert; bei gewöhnlicher Temperatur schwefelgelb, färbt es sich mit zunehmender Temperatur lebhaft rot, um beim Abkühlen wieder die frühere Farbe anzunehmen. Es liegt nahe, diese F'arbenänderung auf eine Umwandlung zweier Modifikationen dieses Salzes zurückzuführen. In der Literatur findet sich jedoch keine Angabe über eine Umwandlungs-

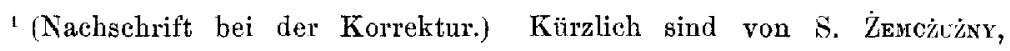
7. anorg. Chem. 57 (1908), 267, Schmelzen von Kaliumchlorid mit Kaliumchromat bzw. mit Kaliumdichromat nach einer besonders empfindlichen Methode (Registrierapparat von Kunnakow) untersucht, worden, wobei auch die Schmelzund Cmwandlungspunkte dieser Chromate genau bestimmt wurden.

2 G. Tamann, Z. anorg. Chem. 37 (1903), 303; 45) (1905), 24; 47 (1905), 294 .

${ }^{3}$ E. Groschuff, Saure Nitrate; $Z$. anorg. Ohem. 40 (1904), 1. 
temperatur. Nach OstwaLD ${ }^{1}$ handeit es sich lediglich um eine Verschiebung des Gebietes, in welchem das Salz die Strahlen des weifsen Lichtes absorbiert, mit der Temperatur, und zwar wandert das Absorptionsgebiet aus dem Violett nach dem Grün, also nach längeren Wellen, wenn die Temperatur steigt.

\section{Umwandlung und Farbenänderung beim Kaliumchromat.}

Bei dem Versuch, die Schmelztemperatur zu bestimmen, fand ich auf den Abkühlungskurven aufser dem Haltepunkt für die Krystallisation der Schmelze $\left(971^{\circ}\right)$ noch einen zweiten sehr deutlichen Haltepunkt bei $666^{\circ}$ (vgl. Tabelle 1 auf S. 106). Da dieser Haltepunkt auch auf der Erhitzungskurve zu beobachten war, liegt eine reversible Lmwandlung zweier krystallisierter Modifikationen des Kaliumchromats vor. Dies bewog mich, auch die Farbenänderung des Kaliumchromats beim Erhitzen näher zu untersuchen.

Ich erhitzte hierzu das Kaliumchromat in einem Reagensrohr zunächst im Wasser-, dann im Salpeterbade auf konstante Temperaturen. Bei $100^{\circ}$ war das Chromat bereits etwas dunkler als bei Zimmertemperatur. Mit steigender Temperatur dunkelte das Chromat weiter nach, zugleich trat immer deutlicher die rote Farbe hervor; bei $250^{\circ}$ war das Chromat etwa apfelsinengelb, bei $400^{\circ}$ wie das Kaliumdichromat gefärbt. Die Zeitdauer des Erhitzens war auf die Farbe ersichtlich ohne Einflufs, Ein plötzlicher an eine bestimmte Temperatur gebundener Farbenwechsel war keinesfalls zu beobachten. Auch macht die Farbenänderung nicht bei der Umwandlung bei $666^{\circ}$ Halt. Die Farbe vertieft sich jenseits desselben mit zunehmender Temperatur bis zu einem ziemlich dunklen Rot. Ein Sprung oder ein Knick macht sich bei der Umwandlung in der Farbenänderung nicht bemerkbar; die durch die Umwandlung etwa hervorgerufene Farbenänderung ist jedenfalls weniger auffällig als die Farbenänderung, welche jede der beiden Modifikationen aufserhalb des Bereiches der Umwandlungstemperatur bei gröfseren Temperaturänderungen erleidet.

Parallel mit der Farbenänderung läuft noch eine zweite Erscheinung, das Dekrepitieren. Untersucht man diese Erscheinung, wie oben die Farbenänderung, so findet man, dafs das Dekrepitieren bei etwa $200^{\circ}$ merklich wird, und, oberhalb der Umwandlung bei $666^{\circ}$ aufhört. Auch wenn man mit dem Erhitzen stufenweise vor-

1 Ostwatd, Grundlinien der anorganischen Chemie, 1900, (1. Aufl.), S. 614. 
schreitet und jedesmal bei konstanter Temperatur so lange erhitzt, bis das Dekrepitieren beendet erscheint, tritt es stets wieder von neuem auf, sobald man die Temperatur steigert. Die Krystalle zeigen erst Risse und Spalten, zerspringen allmählich in kleinere Stücke und zerfallen schlief'slich in ein feinkörniges (krystallinisches) Pulver. Von dem Dekrepitieren verschieden ist das knisternde Geräusch, welches die Umwandlung begleitet, insofern als das Knistern während der Umwandlung nicht nur beim Erhitzen, sondern auch beim Abkühlen regelmälsig bemerkbar ist, das Dekrepitieren zwischen 200 und $666^{\circ}$ aber nur beim Erhitzen von aus wässeriger Lösung erhaltenen Krystallen erfolgt. Wiederholt man das Erhitzen mit demselben Präparat von Zimmertemperatur ausgehend, so beobachtet man, wenn das Präparat bereits das erstemal über $666^{\circ}$ hinaus erhitzt war, nur das die Umwandlung bei $666^{\circ}$ begleitende Knistern. Es liegt nahe, das Dekrepitieren zwischen 200 und $666^{\circ}$ auf eingeschlossene Spuren Mutterlauge zurückzuführen. In der Tat konnte auch beim Kaliumchromat das Austreten von Wasser beobachtet werden. In dem vorliegenden Fall überrascht besonders die Höhe der Temperatur, bei welcher noch Mutterlauge zurückbleibt. Andauernd auf ca. $400^{\circ}$ erhitztes Kaliumchromat verlor beim Erhitzen auf böhere Temperatur noch deutlich nachweisbare Spuren Wasser unter lautem Dekrepitieren. Es scheint, als ob erst bei der durch die Umwandlung bei $666^{\circ}$ erfolgenden gänzlichen Zertrümmerung des Krystallbaues die letzten Reste des eingeschlossenen Wassers entweichen. Auch beim Kaliumsulfat beobachtet man ähnliche auffällige Dekrepitationserscheinungen; die Menge der eingeschlossenen Mutterlauge pflegt bei diesem Salz weniger auffällig als bei dem Chromat zu sein. Diese Beispiele zeigen von neuem, wie schwer es ist, eingeschlossene Wasserspuren aus Krystallen zu entfernen, eine Tatsache, auf welche TH. W. RIOHards ${ }^{1}$ bei seinen Atomgewichtsuntersuchungen wiederholt aufmerksam gemacht hat.

Die neuere Krystallographie hat mehrfach gezeigt, dal's bei manchen Salzen Di- und Polymorphie erst bei den Mischkrystallen hervortreten. Durch Anwendung der thermischen Analyse fand BoskE ${ }^{2}$ bei den Natriumsalzen der Schwefelsäuregruppe eine grofse Zahl von Modifikationen mit verwickelten Isomorphiebeziehungen. Es schien mir nicht ausgeschlossen, dafs sich hinter den oben be-

1 TH. W. Richands, Vortrag, Ber. deutsch. chem. Ges. 40 (1907), 2770.

" Boere, Z. anorg. Chem. 50 (1906), 355. 
schriebenen Krscheinungen eine Neigung zur Bildung weiterer Modifikationen des Kaliumchromats versteckt. Ich habe deshalb noch das Verhalten des Kaliumchromats zu einem isomorphen Salze, und zwar zu Kaliumsulfat, zu studieren gesucht.

\section{Das System Kaliumchromat-Kaliumsulfat.}

Es ist schon lange ${ }^{1}$ bekannt, dafs Kaliumchromat und Kaliumsulfat, aus wässeriger Lösung gewonnen, beide rhombisch krystallisieren und miteinander isomorph sind. Die Isomorphie ist ziemlich weitgehend, entspricht aber doch nicht röllig dem idealsten Fall. Nach L. Stibing ${ }^{2}$ läfst sich nämlich die Grölse der topischen Achsen der Mischkrystalle nicht nach der einfachen Mischungsregel berechnen. Nach optischen Beobachtungen von K. MaLLaRD ${ }^{3}$ ist das Kaliumsulfat dimorph; das bei gewöhnlicher Temperatur rhombische Salz geht zwischen 600 und $650^{\circ}$ in eine hexagonale Modifikation über. Le Chatelizk ${ }^{4}$ fand auf thermischem Wege $550^{\circ}$ als Umwandlungstemperatur. Hütrner und TamMaxin ${ }^{5}$ geben neuerdings $587^{\circ}$ an. Über die Isomorphie des Kaliumchromats und -sulfats bei höherer Temperatur ist noch nichts bekannt.

Der Schmelzpunkt des Kaliumsulfats ist öfter ${ }^{6}$ bestimmt worden, zuletzt von Hüttrer und Tammane, ${ }^{5}$ welche als Schmelzpunkt $1074^{\circ}$ fanden. Den Schmelzpunkt des Kaliumchromats gibt Le Chatelier zuerst ${ }^{4}$ zu $975^{\circ}$, später ${ }^{7}$ zu $940^{\circ}$ an.

Das angewandte Versuchsverfahren schlols sich im wesentlichen der im Göttinger Institut für anorganische Chemie ausgearbeiteten thermischen Analyse an. Kaliumsulfat und Kaliumchromat, welche wegen des lästigen Dekrepitierens zuror über die Umwandlung bei $666^{\circ}$ hinaus erhitzt worden waren, wurden in Porzellangefärsen von Reagensglasform in einer Gesamtmenge von $20.0 \mathrm{~g}$ zusammengeschmolzen. Nach gutem Umrühren wurde die Abkühlungs- und

'Mitschenlich, Pogg. Ann. 15 (1830), 168. - Brooke, Ann. d. phil. 22,120 .

${ }^{2}$ Strbing, Z. f. Krystall. 41 (1906), 611.

3 Vergl. O. Lenuane, Molekular-Physik, Bd. 1, S. 171, (Leipzig 1888). Groth, Einleitung in die chem. Krystall, S. 6, (Leipzig 1904).

${ }^{4}$ Le Chatelier, Bull. soc. chim. [2] 47 (1887), 300.

5 Hütriner und Tamanan, Z. anorg. Chem. 43 (1904), 215.

6 Vergl. Landolt-Börsstein, Tabellen, 3. Aufl, S. 275.

7 Le Chatelier, Compt. rend. 118 (1894), 350. 709. 800. 
die Erhitzungskurve je zweimal aufgenommen. Zur Temperaturmessung diente ein an der Physikalisch-Technischen Reichsanstalt geeichtes LE Chatedier sches Thermoelement, welches durch Bestimmung des Schmelzpunktes des Kaliumsulfats öfters kontrolliert wurde. Die Ablesungen erfolgten in der Regel alle 10 Sekunden. Das Thermoelement war dabei durch ein dünnwandiges Porzellanrohr vor der Berührung mit der Schmelze geschützt. Die kalte Lötstclle wurde auf $0^{\circ}$ gehalten, wodurch eine besondere Korrektion für die Temperatur derselben überflüssig wird. Als Heizquelle dienten selbstgefertigte elektrische Öfen: Mit einem Boden versehene eiserne Röhren von etwa $12 \mathrm{~cm}$ Länge und $2 \mathrm{~cm}$ innerem Durchmesser wurden mit etwa drei Lagen Asbestpapier umgeben, und dann mit etwa $8.8 \mathrm{~m}$ Nickeldraht von $0.5 \mathrm{~mm}$ Durchmesser spiralförmig umwickelt. Die Zwischenräume zwischen den Spiralen, sowie den einzelnen Asbestlagen wurden mit einem feinen Tonbrei ausgefüllt zur Verbesserung der Isolation, insbesondere auch gegen das Eisenrohr. Nach dem Trocknen kam das Rohr in einen Tonzylinder von etwa $7 \mathrm{~cm}$ Durchmesser, während der Zwischenraum mit Magnesia usta ausgefullt und oben mit Asbestpappe abgeschlossen wurde. Dieser Ofen hat sich ausgezeichnet für die Versuche bewährt. Es können leicht und schnell Temperaturen bis zu etwa $1200^{\circ}$ erreicht werden. Die Erhitzung ist sehr gleichmälsig, so dal's die darin befindlichen Porzellanröbren auch bei raschem Firhitzen nicht springen.

In Tabelle 1 sind die Resultate der Abkühlungskurven zusammengestellt und in Fig. 1 graphisch dargestellt. Das Ende der

Tabelle 1.

System Kaliumsulfat-Kaliumchromat.

\begin{tabular}{|c|c|c|c|c|c|c|c|}
\hline \multirow{2}{*}{\multicolumn{2}{|c|}{$\begin{array}{c}\text { Gebalt an } \\
\mathrm{K}_{2} \mathrm{SO}_{4}\end{array}$}} & \multicolumn{3}{|c|}{ Krystallisation } & \multicolumn{3}{|c|}{ Umwandlung } \\
\hline & & \multirow{2}{*}{$\begin{array}{l}\text { 'Temp. } \\
\text { in }{ }^{\circ} \mathrm{C}\end{array}$} & \multirow{2}{*}{$\begin{array}{l}\text { 7eit- } \\
\text { dauer } \\
\text { in Sek. }\end{array}$} & \multirow{2}{*}{$\begin{array}{l}\text { Abkühlungs- } \\
\text { geschwindig- } \\
\text { keit in o } 0 \\
\text { pro Sek. }\end{array}$} & \multirow{2}{*}{$\begin{array}{l}\text { Temp. } \\
\text { in } 0 \mathrm{C}\end{array}$} & \multirow{2}{*}{$\begin{array}{l}\text { Zeit- } \\
\text { dauer } \\
\text { in Sek. }\end{array}$} & \multirow{2}{*}{$\begin{array}{l}\text { Abkühlungs- } \\
\text { geschwindig- } \\
\text { keit in } 0 \\
\text { pro Sek. }\end{array}$} \\
\hline $\begin{array}{c}\text { Gew.- } \\
\% \\
\end{array}$ & $\begin{array}{c}\text { Mol.- } \\
\%\end{array}$ & & & & & & \\
\hline$\left(\mathrm{K}_{2} \mathrm{CrO}_{4}\right.$ & & & & & & & \\
\hline 0 & 0 & 971 & 160 & 0.65 & 666 & 110 & 0.28 \\
\hline 20 & 21.8 & $975-965$ & 130 & 0.63 & $652-644$ & 110 & 0.27 \\
\hline 40 & 42.6 & $996-989$ & 115 & 0.67 & $640-637$ & 90 & 0.28 \\
\hline 60 & 62.6 & $1017-1011$ & 115 & 0.65 & $625-624$ & 90 & 0.23 \\
\hline 80 & 81.7 & $1038-1032$ & 125 & 0.65 & 605 & 100 & 0.25 \\
\hline$\left(\mathrm{K}_{3} \mathrm{SO}_{4}\right)$ & & & & & & & \\
\hline 100 & 100.0 & 1072 & 125 & $0.6 \dot{3}$ & 586 & 110 & 0.25 \\
\hline
\end{tabular}


Krystallisations-, resp. Umwandlungsintervalle ist nach der Methode von Tammañ ${ }^{1}$ berechnet, welche auf einem Vergleich der Ab. kühlungskurven des untersuchten Gemisches und der reinen Komponenten beruht. Da die Erhitzungskurven etwas weniger gleich-

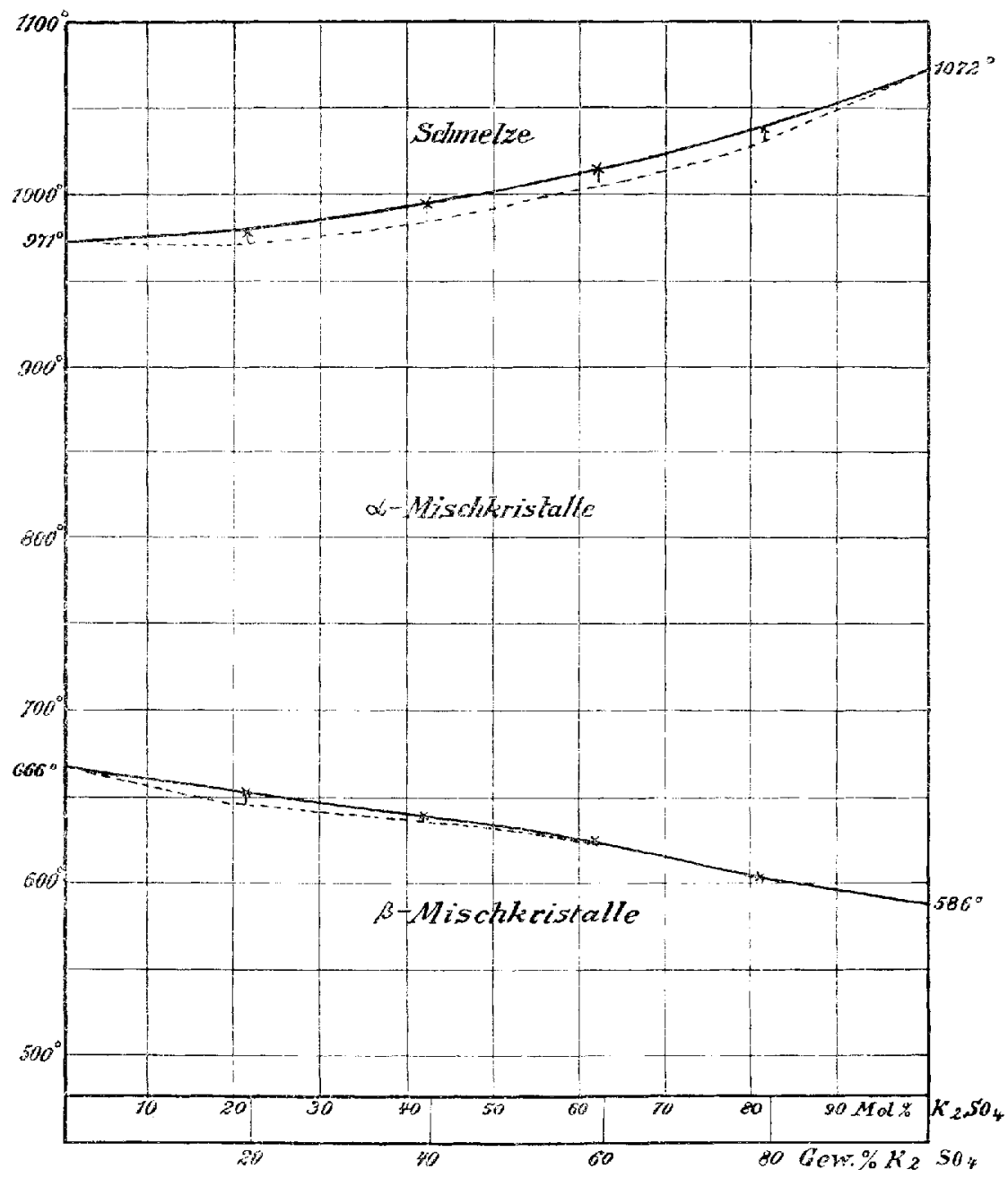

Fig. 1. Zustandsdiagramm Kaliumsulfat-Kaliumchromat.

mälsig ausgebildet waren als die Abkühlungskurven, sind sie hier nicht berücksichtigt worden; doch ergaben sie fast die gleichen Resultate wie diese.

1 G. Tammane, Z. anorg. Ohem. $47(1905), 136$. 
Wie das Zustandsdiagramm in Fig. 1 zeigt, liegen sehr einfache Verhältnisse vor. Kaliumchromat und Kaliumsulfat bilden sowohl bei der Krystallisation aus dem Schmelzflufs (im $\alpha$-Zustand), als anch nach der Umwandlung (im $\beta$-Zustand) eine kontinuierliche Reihe von Mischkrystallen ohne Maximum oder Minimum in der Schmelz- bzw. Umwandlungskurve. Es sind daher nicht nur die bei gewöhnlicher Temperatur existierenden $\beta$-Formen, sondern auch die $\alpha$-Formen der beiden Salze miteinander isomorph; während die $\beta$-Mischkrystalle rhombisch sind, dürften die $\alpha$-Mischkrystalle hexagonal sein. Das Kaliumchromat besitzt eine starke Färbekraft; Mischkrystalle mit $20 \%$ Kaliumchromat erschienen noch schwefelgelb. Die Farbeänderungen, welche bei reinem Kaliumchromat beobachtet wurden, zeigten sich auch bei den Mischungen. Neue Modifikationen wurden nicht aufgefunden.

\section{Das System Kaliumchromat-Chromsäure.}

\section{Die Chromsäure.}

Nach Angaben in der Literatur soll sich Chromsäure erst nach dem Schmelzen zersetzen. Als Zersetzungstemperaturen finden sich angegeben oberhalb $250^{\circ},{ }^{1}$ suwie oberhalb $200^{\circ 2}$. Nach Traube ${ }^{3}$ schmilzt die Chromsäure bei $180-190^{\circ}$, nach Morssan ${ }^{4}$ erstarrt sie bei ca. $170^{\circ}$. Nach ZeTrNow š lälst sich die Chromsäure bei vorsichtigem Erhitzen unzersetzt schmelzen, und beginnt bei $170^{\circ} \mathrm{zu}$ erstarren unter Temperaturerhöhung auf $192-193^{\circ}$ während des Krystallisierens. Es schien danach vielleicht noch möglich, das Schmelzdiagramm des Systems Chromsäure-Kaliumchromat aufzunehmen.

Chromsäure, Präparat „Kahlbaum", wurde in Glasgefälsen im elektrischen Nickeldrahtofen geschmolzen und die Abkühlungskurve mit einem in der Reichsanstalt geeichten Thermoelement aus Kupfer-Konstantan aufgenommen. Je nach dem Grade der Unterkühlung wurden verschiedene Krystallisationstemperaturen erhalten.

1 Roscoe-Sohormemane, Ausfïbrliches Jehmbuch der Chemic, 2. Bd., (2. Aufl, 1888-89), S. 595, und andere Lehrbücher.

Morsan, Traité de chimie minérale, Bd. IV, S. 620.

3 Tratbe, Lieb. Ann. 66 (1848), 165.

${ }^{4}$ Morssan, Ann. chim. phys. [6] 5 (1885), 468.

5 Zetrow, Pogg. Ann. 143 (1871), 474. 
Die Chromsäure ist bei diesen Temperaturen bereits zähfliussig, und die Krystallisationsgeschwindigkeit offenbar schon zu gering, so dafs die wahre Schmelztemperatur nach Beginn der spontanen Krystallisation nicht mehr erreicht werden konnte. Unterkühlungen wurden bis auf $170^{\circ}$ herab erbalten; die Krystallisationstemperatur war dann $180-183^{\circ}$. Beim Erhitzen ergab sich als Schmelzpunkt $196^{\circ}$. Bei etwa $200^{\circ}$ machte sich bereits Zersetzung bemerkbar durch schwachen Geruch nach Ozon und Auftreten einzelner kleiner Gasblasen. Hielt man die Temperatur einige Zeit auf $200^{\circ}$, wurde infolge der Zersetzung eine etwas niedrigere Schmelztemperatur gefunden; auch die Fähigkeit zur Unterkühlung vergrörserte sich dann. ${ }^{1}$ Es ist nicht ausgeschlossen, dals die wahre Schmelztemperatur der Chromsäure noch etwas höher liegt als bei $196^{\circ}$.

Die Hoffnung, dals die Chromsäure auf Zusatz von Kaliumchromat erheblich beständiger werden könnte, wurde nicht erfüllt. Die Chromsäure liels sich weder mit Kaliumchromat noch mit Kaliumdichromat ohne erhebliche Zersetzung zusammenschmelzen. Auch Kaliumtrichromat, welches nach SoHrirn EMakens ${ }^{2}$ aus einer Lösung von Kaliumdichromat und Chromsäure in Wasser dargestellt war, zersetzt sich beim Schmelzen. Es wurde deshalb darauf verzichtet, Systeme mit mehr als $50 \mathrm{Mol} .^{\circ} \% \mathrm{CrO}_{3}$ zu untersuchen. Dagegen lie؟s sich leicht das Schmelzdiagramm für Kaliumdichromat-Kaliummonochromat aufnehmen.

\section{Das System Kaliumdichromat Kaliummonochromat.}

Kaliumdichromat und Kaliummonochromat wurden in Porzellangefäfsen zusammengeschmolzen. Die Resultate der Abkühlungskurven sind in Tabelle 2 und in Fig. 2 zusammengestellt. Die Zeitdauer der Umwandiung ist in Senkrechten (mit willkürlicher Einheit für die Zeit) auf der Umwandlungslinie, die der eutektischen Krystallisation auf der eutektischen Horizontalen aufgetragen. Aus den Erhitzungskurven war das Ende der Schmelzung meist nicht gut zu ermitteln. Das Eutektikum $\mathrm{K}_{2} \mathrm{Cr}_{2} \mathrm{O}_{7}+\mathrm{K}_{2} \mathrm{CrO}_{4}$ liegt bei ca. $393^{\circ}$ und ca. 99 Gew.- $\% \mathrm{~K}_{2} \mathrm{Cr}_{2} \mathrm{O}_{7}$, also dem Schmelzpunkt des reinen Kaliumdichromats, $396^{\circ}$, sehr nahe. Die Umwandlung des

1 Bei längerem Erhitzen (6 Stunden) im zugeschmolzenen evakuierten Glasrohr konnte auch an krystallisierter Chromsäure unterhalb der Schmelztemperatur (bei $150-160^{\circ}$ ) Zersetzung derselben beobachtet werden.

2 Schreinemakers, Zeitschr. phys. Chem. 55 (1906), 85; vergl. auch Koppeta und Blumentual, Z. anorg. Chem. 53) (1907), 228. 
Tabelle 2.

System Kaliumchromat-Chromsäure (bzw. Kaliumdichromat).

\begin{tabular}{|c|c|c|c|c|c|c|c|c|}
\hline \multicolumn{3}{|c|}{ Gehalt an } & \multirow{2}{*}{$\begin{array}{l}\text { Abkiüblungs- } \\
\text { geschwindig- } \\
\text { keit in }{ }^{\circ} \mathrm{C} \\
\text { pro Sek. }\end{array}$} & \multirow{2}{*}{$\begin{array}{l}\text { 'lemp. } \\
\text { d. ersten } \\
\text { Knick } \\
\text { in }{ }^{0} \mathrm{C}\end{array}$} & \multicolumn{2}{|c|}{ Umwandlung } & \multicolumn{2}{|c|}{ Eutekt. Krystall. } \\
\hline $\begin{array}{c}\mathrm{K}_{2} \mathrm{Cr}_{2} \mathrm{O}_{7} \\
\text { Gew.- } \\
\%\end{array}$ & $\begin{array}{c}\mathrm{Cr} \\
\text { Gew.- } \\
\%\end{array}$ & $\begin{array}{c}\mathrm{O}_{3} \\
\text { Mol.- } \\
\%\end{array}$ & & & $\begin{array}{l}\text { Temp. } \\
\text { in }{ }^{\circ} \mathrm{C}\end{array}$ & $\begin{array}{l}\text { Zeit- } \\
\text { dauer } \\
\text { in Sek. }\end{array}$ & $\begin{array}{l}\text { Temp. } \\
\text { in } 0\end{array}$ & $\begin{array}{l}\text { Zeit- } \\
\text { dauer } \\
\text { in Sek. }\end{array}$ \\
\hline $\begin{array}{c}\left(\mathrm{K}_{2} \mathrm{CrO}_{4}\right) \\
0\end{array}$ & 0 & 0 & 0.65 & $\begin{array}{c}971 \\
\text { (Zeitd.160) }\end{array}$ & 666 & 110 & - & - \\
\hline 25.0 & 8.5 & 15.8 & 0.55 & 909 & 665 & 80 & 390 & 175 \\
\hline 50.0 & 17.0 & 28.4 & 0.35 & 812 & 665 & 40 & 393 & 280 \\
\hline 75.0 & 85.5 & 39.9 & 0.23 & 675 & 665 & 10 & 393 & 400 \\
\hline 90.0 & 30.6 & 46.1 & 0.21 & 504 & - & - & 394 & 450 \\
\hline 95.0 & 32.3 & 48.1 & 0.18 & 444 & - & - & 392 & 460 \\
\hline 97.5 & 83.1 & 49.0 & 0.17 & 414 & - & - & 393 & 495 \\
\hline $\begin{array}{r}98.75 \\
\left(\mathrm{~K}_{z} \mathrm{Cr}_{z} \mathrm{O}_{7}\right)\end{array}$ & 33.6 & 49.5 & 0.15 & 402 & - & - & 394 & 495 \\
\hline 100.0 & 34.0 & 50.0 & 0.22 & $\begin{array}{c}396 \\
\text { (Zeitd.495) }\end{array}$ & - & - & - & - \\
\hline
\end{tabular}

$\alpha-\mathrm{K}_{2} \mathrm{CrO}_{4}$ in $\beta-\mathrm{K}_{2} \mathrm{CrO}_{4}$ erfolgt in Berührung mit einer Schmelze, die ca. 75.5 Gew. $-\% \quad \mathrm{~K}_{2} \mathrm{Cr}_{2} \mathrm{O}_{7}$ enthält. Die Schmelzkurve für Kaliummonochromat zerfällt demgemärs in zwei Teile, welche sich ohne wesentlichen Knick fortsetzen.

Nach E. Mrtscherlich ${ }^{1}$ krystallisiert Kaliumdichromat aus der Schmelze in derselben (triklinen) Form, wie bei gewöhnlicher 'T'emperatur aus wässeriger Lösung, zerfällt aber beim Abkühlen bei einer bestimmten Temperatur zu einem Pulver. Nach Mrtsceeruich rührt das Zerfallen des geschmolzenen Salzes von einer ungleichen Zusammenziehung der Krystalle nach verschiedenen Richtungen her. Nach G. Tammann ${ }^{2}$ liegt aber eine Umwandlung mit unmerklicher Energieänderung bei grofser Volumenänderung vor; die Abküblungskurve zeigte keine Diskontinuität, während das Probierglas bei $240^{\circ}$ infolge der die Umwandlung begleitenden Ausdehnung gesprengt wurde. Ich fand auf den Abkühlungskurven ebenfalls keinen Knick. ${ }^{3}$

1 E. Mrrscherlich, Pogg. Ann. 28 (1833), 120.

${ }^{2}$ G. Tammane, Krystallisieren und Schmelzen, (Leipzig 1903), S. 40.

3 (Nachschrift bei der Korrektur.) S. Żemcżuży, Z. anorg. Chem. 56 (1908), 273, ist es inzwischen mittels eincr empfindlichcren Methode (Registrierapparat von Kurnakow und ungeschütztes Thermoelement) gelungen einen deutlich ausgesprochenen Haltepunkt bei $236^{\circ}$ aufzufinden. 


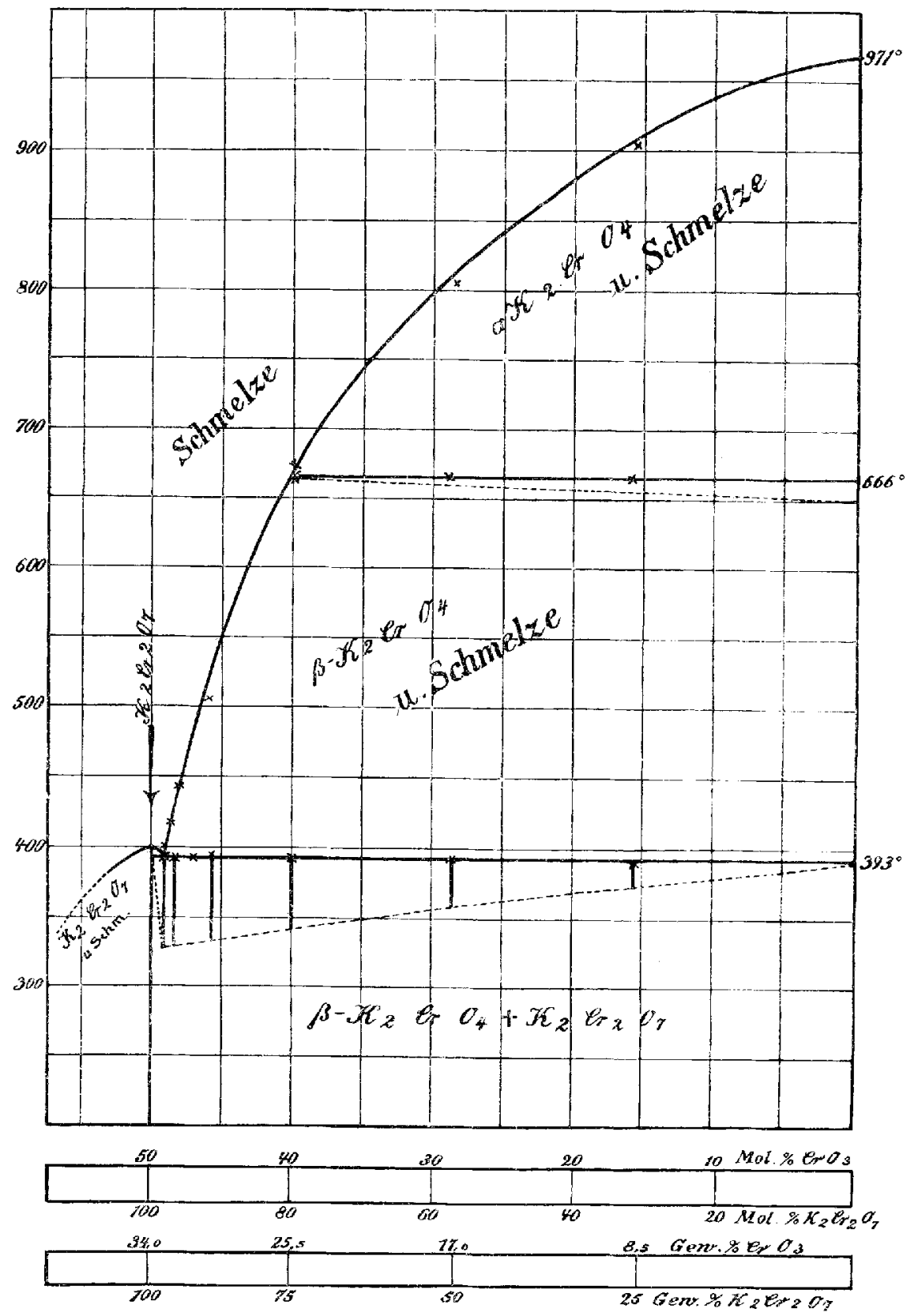

Fig. 2. Zustandsdiagramm Kaliumchromat-Kaliumdichromat (ChromsäureKaliumchromat). 
Durch die Ausdehnung wurden selbst Porzellangefäfse mit $2 \mathrm{~mm}$ dicken Wänden gesprengt.

Das Kaliumdichromat verträgt hohes Erhitzen auch nicht völlig ohne Zersetzung. Schon BuFr ${ }^{1}$ beobachtete, dals Kaliumdichromat, 30 Minuten unter Luftabschlufs geschmolzen, infolge Abgabe von Sauerstoff $0.02 \%$ an Gewicht verlor; beim Auflösen des Dichromats in Wasser blieb ein kleiner Rückstand von grünem Chromoxyd. Auch ich beobachtete, dals Schmelzen, die anf etwa $1000^{\circ}$ erhitzt waren, geringe Mengen eines schwarzgrünen in Wasser unlöslichen Chromoxyds enthielten; die erstarrten Schmelzen besafsen dann statt einer rotgelben eine ins Bräunliche gehende Farbe. Ein wesentlicher Einflufs dieser Zersetzung auf die Schmelztemperatur konnte jedoch nicht beobachtet werden.

\section{Zusammenfassung.}

1. Kaliummonochromat existiert in zwei krystallinischen Modifikationen, welche sich bei $666^{\circ}$ ineinander umwandeln. Die bei höherer Temperatur existierende $\alpha$-Form bildet mit hexagonalem $\alpha$-Kaliumsulfat, die bei gewöhnlicher Temperatur bestehende rhombische $\beta$-Form, mit $\beta$.Kaliumsulfat eine lückenlose Reihe von Mischkrystallen. Die Schmelz- und die Umwandlungskurven zeigen weder ein Maximum noch ein Minimum. Die Farbenänderung des $\beta$-Kalinmchromats mit der Temperatur erfolgt allmählich und zeigt keine polymorphe Änderung an.

2. Die Chromsäure schmilzt bei ca. $196^{\circ}$ unter geringer Zersetzung; die Schmelze lälst sich leicht um $26^{\circ}$ unterkühlen. Gegen $200^{\circ}$ wird die Zersetzung der Chromsäure schon sehr merklich.

3. Es gelingt nicht, Schmelzen von Chromsäure und Kaliumchromat mit mehr als 50 Mol. $\%_{0} \mathrm{CrO}_{3}$ ohne Zersetzung der Chromsäure herzustellen. Kaliumdichromat zersetzt sich beim Erhitzen auf die Schmelztemperatur des Monochromats $\left(971^{\circ}\right)$ merklich unter Abscheidung niederer Oxyde des Chroms.

4. Schmelzpunkt des Kaliummonochromats $971^{\circ}$, des Dichromats $396^{\circ}$, eutektischer Punkt für $\left(\mathrm{K}_{2} \mathrm{CrO}_{4}+\mathrm{K}_{2} \mathrm{Cr}_{2} \mathrm{O}_{7}\right) 393^{\circ}$ mit einer Schmelze der Zusammensetzung 99 Gew. $\% \mathrm{~K}_{2} \mathrm{Cr}_{2} \mathrm{O}_{7}$. Zusammensetzung der Schmelze bei der Umwandlung $\alpha-\mathrm{K}_{2} \mathrm{CrO}_{4} \underset{-}{\longrightarrow}-\mathrm{K}_{2} \mathrm{CrO}_{4}$ bei $666^{\circ}$ ca. 75.5 Gew. $\% \mathrm{~K}_{2} \mathrm{Cr}_{2} \mathrm{O}_{7}$.

1 Burf, Ann. d. Chem. $110(1859), 257$.

Charlottenburg, im Dezember 1907.

Bei der Redaktion eingegangen am 17. März 1908. 